\title{
Frequency Tunability and Slow-Wave Characteristics of a High-Efficiency Ridged MILO
}

\author{
Xiaoyu Wang1,2, Baoping Yang², Xu Xu², Yuwei Fan'1, Ting Shu1 ${ }^{*}$, Ankun Li1, Zeyang Liu1, \\ Xiaomin Wang', Bing Han² \\ ${ }^{1}$ College of Advanced Interdisciplinary Studies, National University of Defense Technology, Changsha, China \\ ${ }^{2}$ NCO School, Space Engineering University, Beijing, China \\ Email: *ting.shu@nudt.edu.cn
}

How to cite this paper: Wang, X.Y., Yang, B.P., Xu, X., Fan, Y.W., Shu, T., Li, A.K., Liu, Z.Y. and Wang, X.M. and Han, B. (2020) Frequency Tunability and Slow-Wave Characteristics of a High-Efficiency Ridged MILO. Journal of Computer and Communications, 8, 91-101.

https://doi.org/10.4236/jcc.2020.812009

Received: October 16, 2020

Accepted: December 17, 2020

Published: December 24, 2020

\begin{abstract}
A high-efficiency ridged magnetically insulated transmission line oscillator (RMILO) is proposed and investigated theoretically and numerically in this paper. In the RMILO, ridge-disk vanes are introduced to enhance the power efficiency. Theoretical investigation shows that the ridge-disk can enhance the coupling impedance of the slow-wave structure (SWS), and so enhance the power efficiency. Moreover, the ridge has a weak influence on frequency, so, it influences little on the tunability of the MILO. In simulation, when the applied voltage is increased to $807 \mathrm{kV}$, the RMILO can get the $3 \mathrm{~dB}$ tunable frequency range with $7.6-13.9 \mathrm{GHz}$ and the $3 \mathrm{~dB}$ tuning bandwidth with $58.6 \%$ which has an increase of $27.6 \%$ compared with the conventional MILO. So, the tuning performance of the RMILO is more superior. Besides, the RMILO gets the maximum output power of $7.1 \mathrm{GW}$, the corresponding power efficiency is $22.6 \%$ and the frequency is $1.400 \mathrm{GHz}$. Furthermore, when the applied voltage is increased to $807 \mathrm{kV}$, high-power microwave with a power of $13.5 \mathrm{GW}$, frequency of $1.400 \mathrm{GHz}$, and efficiency of $24.5 \%$ is generated, which has an increase of $20.2 \%$ compared with the conventional MILO. The simulation results confirm the ones predicted by theoretical analysis.
\end{abstract}

\section{Keywords}

Magnetically Insulated Transmission Line Oscillator, Slow Wave Structure, High Conversion Efficiency

\section{Introduction}

Magnetically insulated transmission line oscillator (MILO) has been considered 
as one major hotspot in the field of high power microwave (HPM) source research because of the advantages of high microwave power, no magnetic field and compact configuration [1]-[7]. The self-magnetic property of the MILO indicates the low power efficiency, which restricts its development. Also, conventional MILO (CMILO) belongs to a typical narrow-band HPM device. It cannot adjust it out frequency [2] [3] [7]. Thus, high power efficiency and frequency tunability are becoming two important directions in the research on MILO [8] [9] [10].

A high-efficiency MILO with ridged disk-loaded vanes has been investigated in our previous studies [3]. When the applied voltage and current are $566 \mathrm{kV}$ and $591.8 \mathrm{kA}$ respectively in simulation, the ridged MILO can generate HPM with the output power of $6.0 \mathrm{GW}$ and the frequency of $1.48 \mathrm{GHz}$. Moreover, the power efficiency reaches up to $21.0 \%$, which has an increase of $19 \%$ compared with the conventional MILO without ridges. In [3], the frequency tunability was not considered. Besides, given the RF breakdown, we restrict the applied voltage and input power of the ridged MILO less than $600 \mathrm{kV}$ and $35 \mathrm{GW}$, respectively. In this sense, the potential to further improve power efficiency is exploited. However, the frequency tunability and high efficiency are two important research directions for the HPM devices.

In this paper, the theoretical analysis of the ridged SWS and the frequency tunability of the ridged MILO are discussed, and the simulation investigation is performed. The simulation results confirm the ones predicted by theoretical analysis.

This paper is organized as follows. The slow-wave characteristics of the conventional slow-wave structure (CSWS) and the ridged slow-wave structure (RSWS) are analyzed to confirm the basic parameters of SWS firstly. Then, the influence of ridge on slow-wave characteristics and coupling impedance is given to further confirm the structure size of the RSWS. Thirdly, the frequency tunability of the RSWS is compared to that of the CSWS. Next, the structure of the RMILO is given and the corresponding frequency tunability and the power efficiency are analyzed. A brief conclusion is drawn finally.

\section{Ridged Slow-Wave Structure}

Conventional slow-wave structure (CSWS) (as shown in Figure 1(a)) is the most common SWSs, but the power efficiency of the conventional MILO is about only $10 \%-15 \%$ [2] [7] [9] [11]. It is confirmed that ridged slow-wave structure (RSWS) (as shown in Figure 1(b)) can improve the power efficiency of HPM devices effectively [3] [12].

RSWS applies the ridges to two sides of the vane, and the inner radii of the ridges and the vanes are the same. The design of this structure is to explore the potential of increasing power efficiency and decreasing the influence on frequency tunability. The dimensions of CSWS and RSWS are presented in Table 1. It should be noted that the only difference between two models is the exist of 


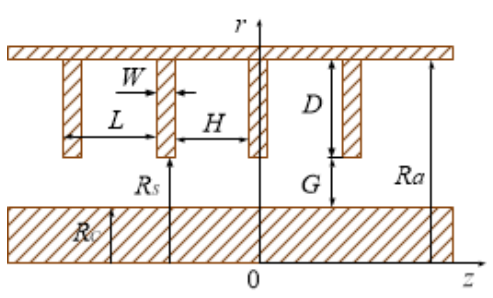

(a)

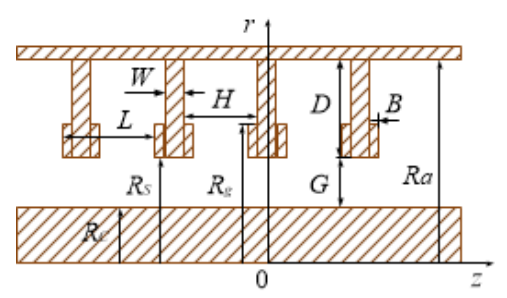

(b)

Figure 1. The model structure of the ridged MILO. (a) CSWS; (b) RSWS.

Table 1. SWS dimensions.

\begin{tabular}{cccc}
\hline$R_{\mathrm{c}} / \mathrm{mm}$ & 30.0 & $L / \mathrm{mm}$ & 28.6 \\
$R_{s} / \mathrm{mm}$ & 49.1 & $H / \mathrm{mm}$ & 22.9 \\
$R_{a} / \mathrm{mm}$ & 96.7 & $D / \mathrm{mm}$ & $49.1-96.7$ \\
$D / \mathrm{mm}$ & 47.3 & $R_{g} / \mathrm{mm}$ & $1.0-11.4$ \\
\hline
\end{tabular}

ridges. The parameters of the ridge are uncertain, which will be confirmed through theoretical analysis in the next section.

The MILO structure and the electron emission are symmetrical azimuthally, so only azimuthal transverse magnetic waves $\left(\mathrm{TM}_{\text {on }}\right)$ exist in CSWS and RSWS when there is beam-wave interaction. Table 1 gives the dispersion curves of RSWS and its comparison with SWS when the applied voltage is $500 \mathrm{kV}$.

The dispersion curves of $\mathrm{TM}_{00}, \mathrm{TM}_{01}, \mathrm{TM}_{02}$ modes of RSWS are described in Figure 2(a), plotting along with the light line and electron beam line. It is noted that the dispersion curves of the three modes are separated with each other by the forbidden frequency bands, which is profitable for the single mode operation. Figure 2(b) gives the comparison of $\mathrm{TM}_{00}$ mode between RSWS and CSWS. The electron beam interacts with the $\mathrm{TM}_{00}$ mode at a frequency of 1.576 $\mathrm{GHz}$ for CSWS, and a frequency of $1.533 \mathrm{GHz}$ for RSWS. Both are around the $\pi$ mode point. Consequently, ridge has a weak influence on frequency.

Besides, the dispersion curve of RSWS is flatter within a large range of axial wave-number $k_{\mathrm{L}}$. This means the ridged MILO has a relatively wider range of operation voltage. It is worth noting that the applied voltage for RMILO, which is not taken into account in our previous studies [3].

\section{Slow-Wave Characteristics Analyses}

The analyses of slow-wave characteristics of RSWS contribute to confirm the parameters of the ridge. With the values in Table 1 and assumed value of ridge width $B=1 \mathrm{~mm}$, the changes of dispersion curves of $\mathrm{TM}_{00}$ mode [Figure 3], normalized phase velocity [Figure 4] and coupling impedance [Figure 5] with the ridge radius $R_{\mathrm{g}}$ are given.

From Figure 3, it is obvious that the dispersion curves of RSWS overlap with $R_{\mathrm{g}}$ in the range of $60-69 \mathrm{~mm}$, so the ridge radius has no influence on the operation frequency of the ridged MILO. 


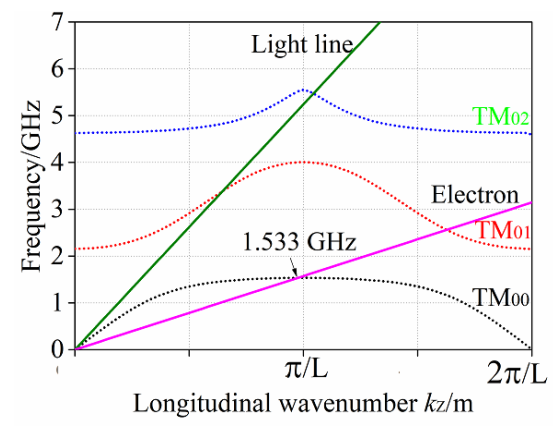

(a)

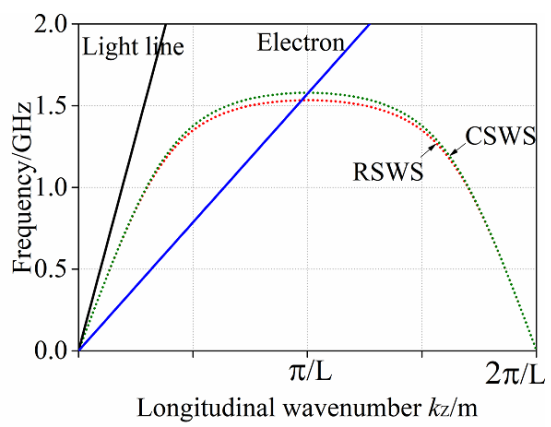

(b)

Figure 2. Dispersion curves of RSWS and SWS. (a) dispersion curves of RSWS; (b) comparison of dispersion curves.

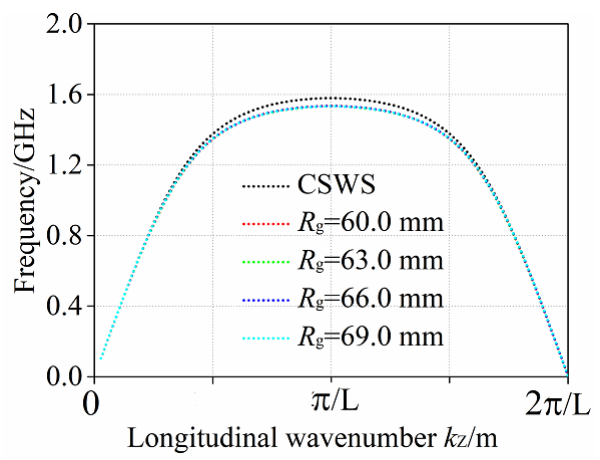

Figure 3. Dispersion curves v.s. $R_{\mathrm{g}}$.

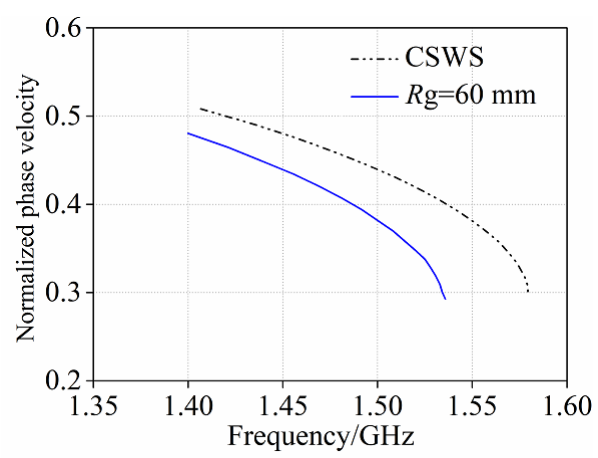

Figure 4. Normalized phase velocity.

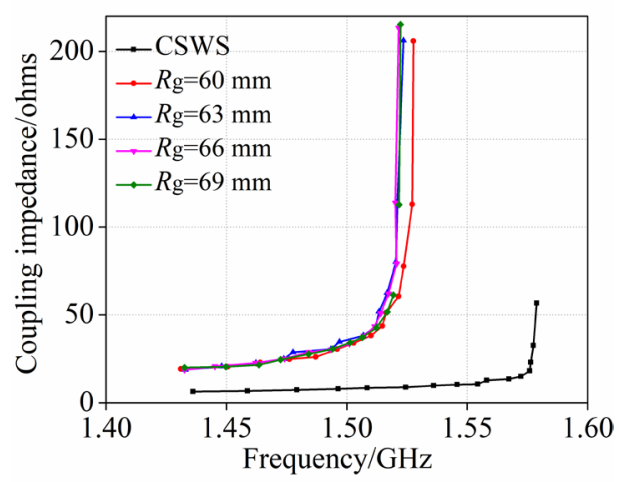

Figure 5. Coupling impedance v.s. $R_{\mathrm{g}}$. 
The coupling impedance $K$ and the normalized phase velocity $v_{\mathrm{pc}}$ can be expressed as [1] [3]

$$
\begin{gathered}
K=\frac{\left(E_{\mathrm{z}}\right)^{2}}{2 \beta^{2} P} \\
v_{\mathrm{pc}}=\frac{v_{\mathrm{p}}}{c}=\frac{\omega}{\beta c}=\frac{2 \pi f}{c \cdot p h a / L}
\end{gathered}
$$

where $E_{\mathrm{z}}$ is the axial electric field, $\beta_{\mathrm{n}}$ is the axial propagation. From Equations (1) and (2), we can obtain $K$ and $v_{\mathrm{pc}}$ respectively. $v_{\mathrm{pc}}$ is only related to the frequency when the SWS is confirmed. Keep the same parameters except the different vg gives the comparison of $v_{\mathrm{pc}}$ of CSWS and RSWS $\left(R_{\mathrm{g}}=60 \mathrm{~mm}\right)$, which demonstrates that the frequency band is narrower and the dispersion flatness is weaker for the RSWS.

Figure 5 describes that the coupling impedance of RSWS is higher during the whole frequency band. Especially, the coupling impedance gets the best value when $R_{\mathrm{g}}=66 \mathrm{~mm}$.

With the values in Table 1 and ridge radius $R_{\mathrm{g}}=66 \mathrm{~mm}$, the impact of the ridge width $B$ is studied. In Figure 6 and Figure 7, the dispersion curve and the frequency are very sensitive to $B$. with the increase of $B$, the dispersion curve is flatter and the frequency is lower.

Figure 8 illustrates the flattest curve of $v_{\mathrm{pc}}$ appears when $B=1$. Figure 9 shows the coupling impedance of RSWS increases with $B$, and is better obviously

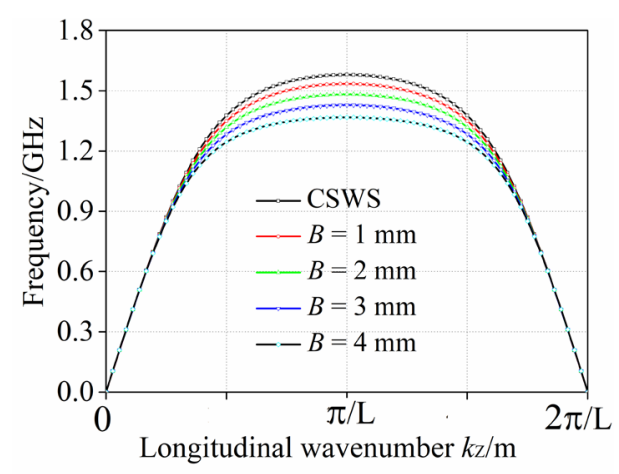

Figure 6. Dispersion curves v.s. $B$.

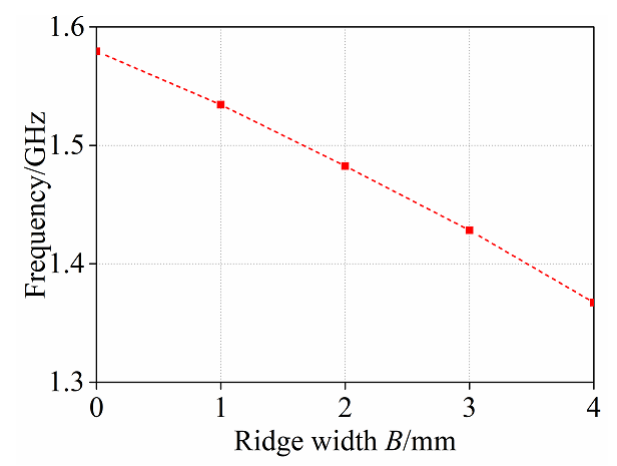

Figure 7. Frequency v.s. $B$. 


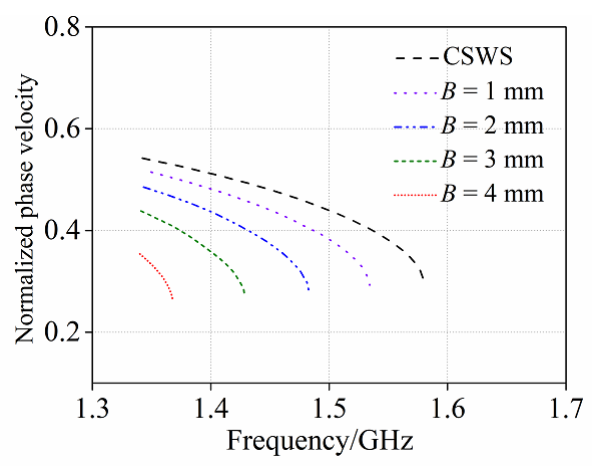

Figure 8. Normalized phase velocity v.s. $B$.

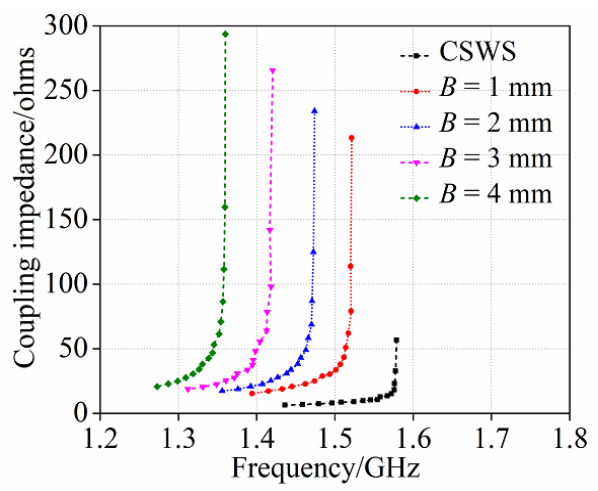

Figure 9. Coupling impedance v.s. $B$.

than that of CSWS. Besides, the coupling impedance tends to be infinitely great with frequency. The reason is that the SWS exists cutoff frequency, so wave over cutoff frequency cannot propagate (namely, $P=0$ ) and coupling impedance becomes infinitely great.

Consequently, the structure of ridge can reinforce the dispersion, but is profitable in enhancing the coupling impedance. In terms of dispersion curves, normalized phase velocity, coupling impedance, etc., the radius and width of the ridge are $R_{\mathrm{g}}=66 \mathrm{~mm}$ and $B=1 \mathrm{~mm}$.

\section{Frequency Tunability of RSWS}

The structure of ridge can affect the frequency of RSWS greatly, so it is necessary to study the frequency tunability of RSWS. Besides, the coupling impedance of RSWS is greater than that of the CSWS, whether the superiority can be continued with frequency tunability still needs study. In this section, the frequency gets adjusted through changing the outer radius of SWS $R_{\mathrm{a}}$.

As shown in Figure 10(a), the dispersion curves get flatter with increase of $R_{\mathrm{a}}$, and the resonant frequency at $\pi$ mode of MILO decreases gradually. In Figure 10(b), when $R_{\mathrm{a}}$ changes the same value, the frequencies at $\pi$ mode of CSWS and RSWS have the similar variation range, which means the ridge cannot influence the frequency tunability of SWS.

Figure 11(a) describes that the frequency coverage falls off with the augment 


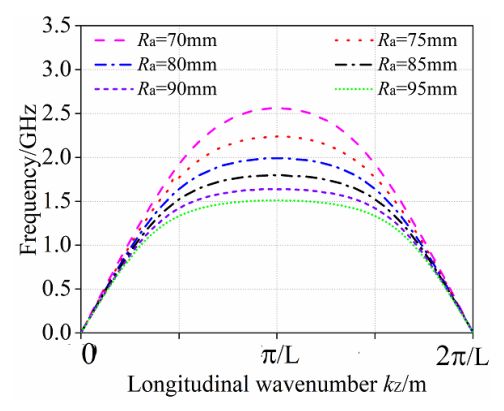

(a)

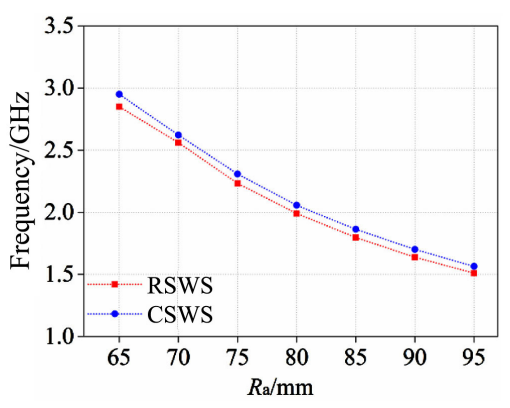

(b)

Figure 10. Comparison of dispersion curves. (a) Dispersion curves at different $R_{\mathrm{a}}$; (b) Frequency at $\pi$ mode v.s. $R_{\mathrm{a}}$.

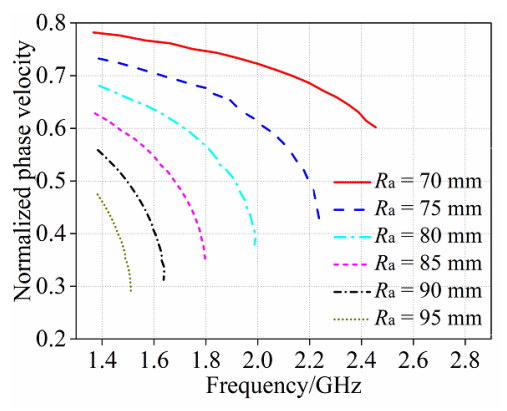

(a)

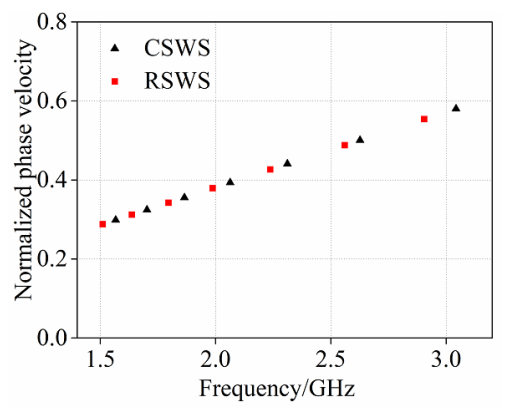

(b)

Figure 11. Comparison of normalized phase velocity. Normalized phase velocities (a) at different $R_{\mathrm{a}}$ (b) v.s. frequency.

of $R_{\mathrm{a}}$ and the curves are downward stepper. Combination of Figure 10(b) and Figure 11(a) can get Figure 11(b). It is obvious that the curves of the normalized phase velocity are nearly lines, which further confirms that the ridge has no substantial impact on the normalized phase velocity.

The frequency at $\pi$ mode varies with the $R_{\mathrm{a}}$, then the coupling impedance of every frequency at $\pi$ mode changes correspondingly. Figure 12 indicates that the coupling impedance of RSWS is higher than that of the CSWS in the whole range of the frequency, proving that the ridge can enhance beam-wave interaction and improve power efficiency of MILO.

\section{Analysis of the Ridged MILO}

With the above analysis, the structure of the ridged MILO (RMILO) is obtained, as shown in Figure 13, which involves the feature structures of focusing electrode, ladder cathode and ridge. Researchers have confirmed that the focusing electrode and ladder cathode contributes to enhance power efficiency [11] [13] [14]. The qualitative physical analysis of ridges in MILO will be described in this section to prove the superiority of the ridge.

Given the ignored potential of frequency tunability and power efficiency in our previous study [3], the proposed MILO has two unique features in terms of the structure compared with the model in [3]. 


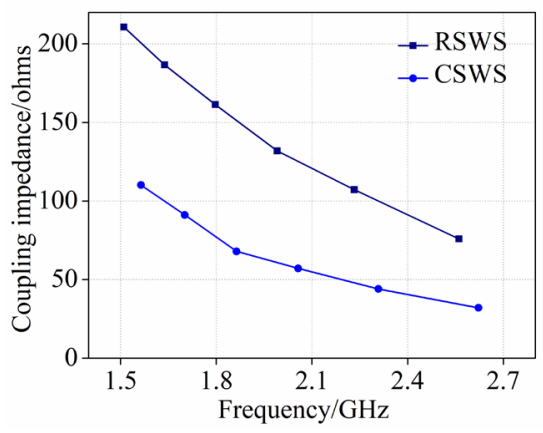

Figure 12. Comparison of coupling impedance.

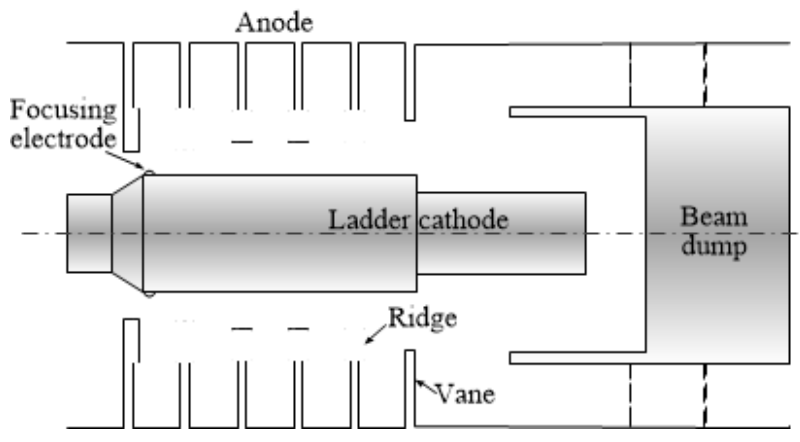

Figure 13. The model structure of the ridged MILO.

1) The inner radii of the SWS vanes and the radius of the cathode under SWS can both increase and decrease, to look for the optimal size, of the model the power efficiency can keep at a high level during frequency tuning.

2) The cathode which is inserted into the beam dump has much less radius and longer length, which can effectively adjust the electric field intensity under the breakdown threshold when the applied voltage is enhanced.

The frequency tunability of the RMILO is analyzed by changing the $R_{\mathrm{a}}$. The comparison of RMILO and the conventional MILO (CMILO) is given in Figure 14. The only difference between the two models is the exist of the ridge.

From Figure 15, the frequency tunability and the power efficiency of the RMILO are superior to that of the CMILO. Specifically, when the frequency is under $1.415 \mathrm{GHz}$, the efficiency of the two models decreases but the curve of the RMILO tends to be mild. Besides, the ridge has little influence on the frequency, which verifies the analysis in section IV. For the RMILO, the $3 \mathrm{~dB}$ tunable frequency range is $7.6-13.9 \mathrm{GHz}$ and the $3 \mathrm{~dB}$ tuning bandwidth is $58.6 \%$. In comparison, the $3 \mathrm{~dB}$ tunable frequency range and bandwidth of the CMILO are $7.9-12.6 \mathrm{GHz}$ and $45.9 \%$, respectively. So, the tuning performance of the RMILO is more superior.

When $R_{\mathrm{a}}=100.0 \mathrm{~mm}$, the RMILO gets the maximum output power of $7.1 \mathrm{GW}$ with the applied voltage of $603 \mathrm{kV}$ and current of $52.2 \mathrm{kA}$. The corresponding power efficiency is $22.6 \%$ and the frequency is $1.400 \mathrm{GHz}$. In comparison, the power efficiency of CMILO under the same condition is $20.1 \%$, which means that the efficiency of the RMILO obtains an increase of $12.4 \%$. 
Setting $R_{\mathrm{a}}=100.0 \mathrm{~mm}$, influence of applied voltage on the performance of the two models is described in Figure 15 and Figure 16. With the increase of the applied voltage, the power efficiency of the two models has the similar variation tendency. However, the efficiency of the RMILO is greater during the whole frequency range. Figure 16 demonstrates that the applied voltage has no effect on the microwave frequency of the device. From Figure 15 and Figure 16, when the applied voltage and input power are $807 \mathrm{kV}$ and $55.1 \mathrm{GW}$ respectively, the RMILO generates HPM with output power of $13.5 \mathrm{GW}$, maximum efficiency of $24.5 \%$, frequency of $1.400 \mathrm{GHz}$. In comparison to the efficiency of $20.7 \%$ in CMILO under the same condition, the efficiency of RMILO has an increase of $20.2 \%$.

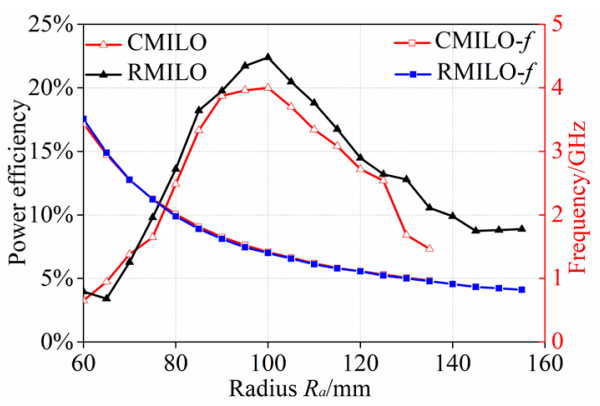

Figure 14. The effect of $R_{\mathrm{a}}$ on RMILO.

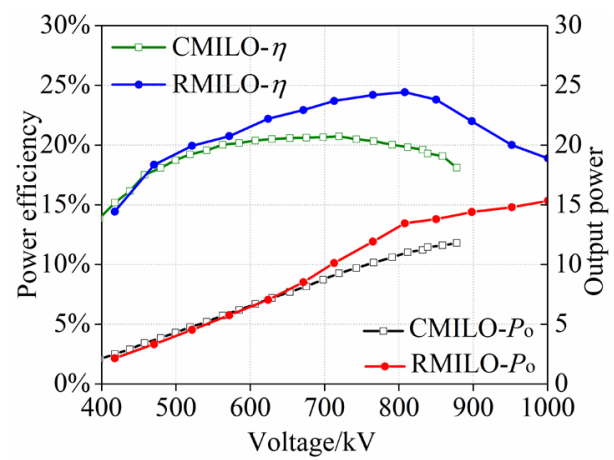

Figure 15. The influence of the applied voltage on efficiency and power.

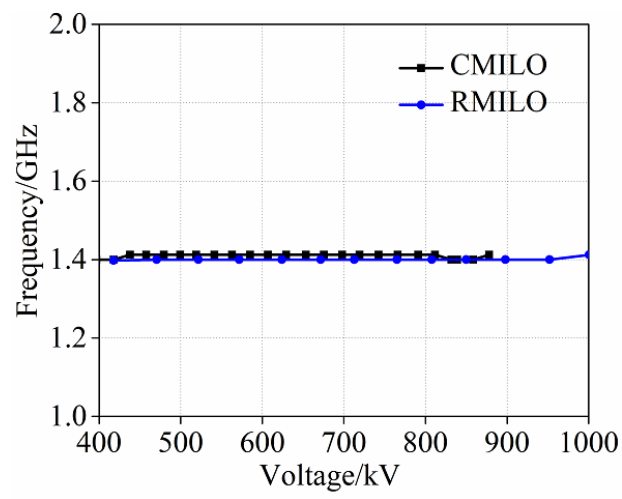

Figure 16. The influence of the applied voltage on frequency. 


\section{Conclusion}

The frequency tunability and the slow-wave characteristics of the RMILO are analyzed. The simulation results show that the structure of ridge contributes to improve the coupling impedance greatly while having no influence on the frequency tunability. The RMILO gets the maximum output power of $7.1 \mathrm{GW}$ with the applied voltage of $603 \mathrm{kV}$ and current of $52.2 \mathrm{kA}$. The corresponding power efficiency is $22.6 \%$ and the frequency is $1.400 \mathrm{GHz}$. Besides, when the applied voltage is increased to $807 \mathrm{kV}$, the RMILO generates HPM with maximum efficiency of $24.5 \%$, which has an increase of $20.2 \%$ compared with CMILO under the same condition.

\section{Acknowledgements}

This work was supported in part in part by the National Natural Science Foundation of China under Grant No. 61671457 and No. 61871390, and in part by the Innovative Team of NCO Teaching on Space Defense and Support, Space Engineering University.

\section{Conflicts of Interest}

The authors declare no conflicts of interest regarding the publication of this paper.

\section{References}

[1] Benford, J., Swegle, J. and Schamiloglu, E. (2007) High Power Microwaves. 3rd Edition, Taylor \& Francis, New York. https://doi.org/10.1201/9781420012064

[2] Wang, X.Y., Fan, Y.W., Shu, T., Li, A.K. and Liu, Z.Y. (2020) Design and Simulation of a Novel High-Efficiency Magnetically Insulated Transmission Line Oscillator. IEEE Trans. Plasma Sci., 47.

[3] Wang, X.Y., Fan, Y.W., Shu, T., Li, A.K., Yu, Y.Q. and Liu, Z.Y. (2019) A High-Efficiency Magnetically Insulated Transmission Line Oscillator with Ridged Disk-Loaded Vanes. IEEE Trans. Plasma Sci, 47, 3974-3977. https://doi.org/10.1109/TPS.2019.2921995

[4] Zhou, J., Liu, D., Liao, C. and Li, Z. (2009) CHIPIC: An Efficient Code for Electromagnetic PIC Modeling and Simulation. IEEE Trans. Plasma Sci, 37, 2002-2011. https://doi.org/10.1109/TPS.2009.2026477

[5] Goplen, B., Ludeking, L., Smithe, D. and Warren, G. (1995) User-Configurable MAGIC for Electromagnetic PIC Calculations. Comput. Phys. Commun., 87, 54-86. https://doi.org/10.1016/0010-4655(95)00010-D

[6] Liu, M., Michel, C., Prasad, S., Fuks, M.I., Schamiloglu, E. and Liu, C.L. (2010) RF Mode Switching in a Relativistic Magnetron with Diffraction Output. Applied Physics Letters, 97, 251501-1-251501-4. https://doi.org/10.1063/1.3529463

[7] Fan, Y.W., Zhong, H.H., Li, Z.Q., Shu, T., Yang, H.W., Yang, J.H., Wang, Y., Luo, L. and Zhao, Y.S. (2008) Investigation of an X-Band Magnetically Insulated Transmission Line Oscillator. Chinese Physics B, 17, 1804-1808. https://doi.org/10.1088/1674-1056/17/5/042

[8] Scott, F.J., Saliba, E.P., Albert, B.J., Alaniva, N., Sesti, E.L., Gao, C.K., Golota, N.C., 
Choi, E.J., Jagtap, A.P., Wittmanc, J.J., Eckardt, M., Harneit, W., Corzilius, B., Sigurdsson, S.T. and Barnes, A.B. (2018) Frequency-Agile Gyrotron for Electron Decoupling and Pulsed Dynamic Nuclear Polarization. Journal of Magnetic Resonance, 289, 45-54. https://doi.org/10.1016/j.jmr.2018.02.010

[9] Fan, Y.W., Wang, X.Y., He, L., Zhong, H.H. and Zhang, J.D. (2015) A Tunable Magnetically Insulated Transmission Line Oscillator. Chinese Physics B, 24, 035203-1035203-4. https://doi.org/10.1088/1674-1056/24/3/035203

[10] Fan, Y.W., Wang, X.Y., Li, G.L., Yang, H.W., Zhong, H.H. and Zhang, J.D. (2016) Experimental Demonstration of a Tunable Load-Limited Magnetically Insulated Transmission Line Oscillator. IEEE Trans. Electron Devices, 63, 1307-1311. https://doi.org/10.1109/TED.2016.2518744

[11] Wang, X.Y. and Fan, Y.W. (2015) Simulational Investigation of a High-Efficiency X-Band Magnetically Insulated Line Oscillator. Plasma Science and Technology, 17, 893-896. https://doi.org/10.1088/1009-0630/16/17/10/14

[12] Yue, L.N., Wang, W.X., Gong, Y.B. and Zhang, K.Q. (2004) Analysis of Coaxial Ridged Disk-Loaded Slow-Wave Structure for Relativistic Traveling Wave Tubes. IEEE Trans. Plasma Sciences, 32, 1086-1092.

https://doi.org/10.1109/TPS.2004.828784

[13] Chen, D.B., Fan, Z.K., Dong, Z.W., Xu, Z., Zhou, H.J., Guo, Y.H., He, H., Gong, H.T. and An, H.S. (2007) Experimental Study on Ladder Cathode L-Band MILO. High Power Laser Part. Beams, 19, 820-824.

[14] Qin, F., Wang, D., Chen, D.B. and Fan, Z.K. (2009) Simulation Investigation of L-Band Ladder Cathode MILO. IEEE Trans. Plasma Sciences, 37, 1921-1924. https://doi.org/10.1109/TPS.2009.2023126 